\title{
Time-delayed 2-Pulse Studies of MALDI Matrix Ionization Mechanisms
}

\author{
Richard Knochenmuss ${ }^{*, \dagger}$ and Akos Vertes* \\ LOC, Universitätsstr. 16, ETH Zentrum, 8092 Zürich, Switzerland, and Department of Chemistry, \\ George Washington University, Washington, D.C., 20052
}

Received: February 7, 2000; In Final Form: April 14, 2000

\begin{abstract}
Individually subthreshold, time-delayed $355 \mathrm{~nm}$ laser pulses were used to investigate matrix-assisted laser desorption/ionization (MALDI) ion formation time scales for the matrix 2,5 dihydroxybenzoic acid. With 30 ps pulses, it was possible to identify a prompt, subnanosecond, mechanism and a slow, nanosecond, process involving at least two steps.
\end{abstract}

\section{Introduction}

Matrix-assisted laser desorption/ionization (MALDI) is, as the name suggests, a composite of two major processes: desorption and ionization. Although considerable progress has been made in understanding the desorption aspect of MALDI, ${ }^{1-13}$ the mechanisms of ionization remain remarkably uncertain. ${ }^{14}$ This is unfortunate because the ion yield in MALDI is quite low, ${ }^{15,16}$ but cannot be rationally optimized until the operative physical and chemical effects are known.

In the case of MALDI with UV lasers, direct 2-photon ionization of the matrix is intuitively attractive and has been observed in special case ${ }^{17}$ but has been shown to be inactive for common matrixes such as benzoic acid derivatives (when using the typical nitrogen laser emitting at $337 \mathrm{~nm}) .{ }^{18,19}$ Although alternatives such as (single photon) excited-state proton transfer ${ }^{20-23}$ have received some attention, it now also appears that this is an unlikely mechanism in the same matrix class. ${ }^{18,19}$

Phenomena such as the matrix suppression effect (MSE) ${ }^{24,25}$ give indications that matrix dimers or aggregates are involved in ionization. The disappearance of all matrix species (radical cations, protonated matrix, and matrix cation adducts) in the MSE suggests that a quasiequilibrium may be approached and that the observed mass spectrum may, under some conditions, be determined more by thermodynamic than kinetic considerations, as has been experimentally found in some cases. ${ }^{26-28}$ Whatever determines the final distribution, though, primary ionization proceeds via transient nonequilibrium processes because the low temperature of the MALDI plume cannot lead to the observed degree of ionization. ${ }^{14}$ Whether the ionization mechanism is exciton pooling, ${ }^{29,18,30}$ proton disproportionation in the nonequilibrium hot electronic ground state, ${ }^{27,31}$ shock waves, $, 9,32$ or a combination of these and others, ${ }^{14}$ observation of the characteristic ion formation time scales could give important clues as to which processes are active.

For relatively long laser pulses, UV-MALDI ion generation has been found to be largely insensitive to the rate at which laser energy is delivered, ${ }^{10,33-37}$ and UV-MALDI has become known as a fluence- rather than irradiance-dependent phenomenon. However, there have been indications that this static

* To whom correspondence should be addressed.E-mail: knochenmuss@org. chem.ethz.ch. Fax: ++4116321292.

$\dagger$ ETH Zürich.

$\doteqdot$ George Washington University. picture is not completely correct. A large dependence of the ion yield on spot diameter has been observed, ${ }^{38}$ with larger spots yielding ions at much lower fluences, even though all sizes showed a similar signal versus fluence behavior above the appearance threshold. This was interpreted in terms of delayed ion generating reactions, in accord with other measurements such as energy deficits ${ }^{39}$ and flight time distributions of MALDI ions. ${ }^{40,41}$ Most importantly, although no irradiance effects were observed down to 550 ps pulse widths, ${ }^{36}$ very significant decreases in mass spectral quality and signal intensity were found for subpicosecond pulses compared to 3 ns pulses. ${ }^{42}$

These results suggest that ion formation times are less than the several-nanosecond pulse widths of typically used lasers (nitrogen or Nd:YAG), and that delayed reactions might be important. One approach to determining these time scales is with a picosecond pump-probe type experiment, as is common in studies of ultrafast chemical dynamics.

Initial two-pulse MALDI (2PM) experiments were reported in connection with the matrix suppression effect. ${ }^{25}$ The $337 \mathrm{~nm}$ beam from a 3 ns nitrogen laser was split, delayed, and recombined on a MALDI sample of valinomycin in 2,5 dihydroxybenzoic acid (DHB). Fixed delays of a few nanoseconds were used to show that nonconcurrent, individually subthreshold pulses could yield excellent MALDI mass spectra. This was a clear indication that energy storage mechanisms are active in UV-MALDI, possibly in the form of excited electronic states.

Subsequent, more detailed, 2PM experiments showed similar effects for analytes in a variety of matrixes. ${ }^{11,43}$ In some matrixes, such as 2,5 DHB and sinapinic acid, analyte ion production decreased exponentially with delay between the laser pulses, with a time constant of about 5 ns. In the case of 2,5 $\mathrm{DHB}$, this corresponds well with the solid-state fluorescence decay time. ${ }^{44,45}$ For some other matrixes, a much slower delay time dependence was found, with a puzzling form. A limitation of these and earlier experiments was that the laser pulse widths were 3-5 ns, which masked any effects that are significantly shorter. It remained quite possible that important structure would emerge at higher time resolution, as we show here.

\section{Experimental Section}

Figure 1 shows a schematic of the experimental setup. A frequency-tripled Nd:YAG laser (Continuum PY-61C, Santa Clara, CA) was used to generate $355 \mathrm{~nm}$ pulses of about $30 \mathrm{ps}$ 


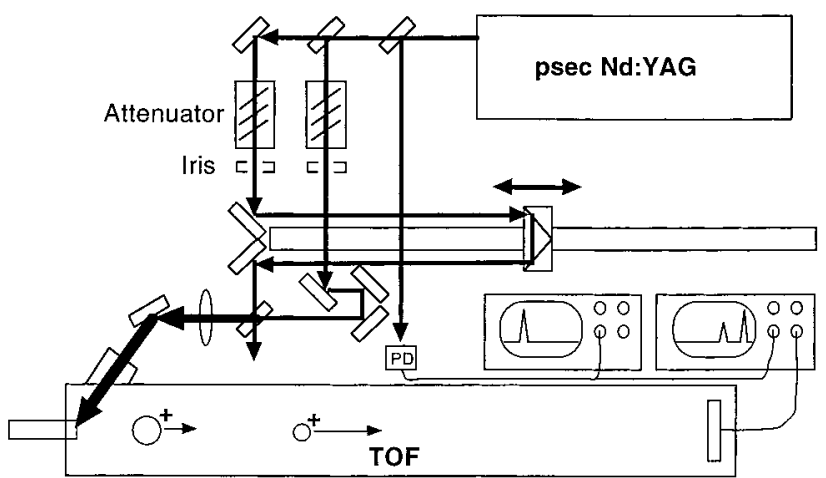

Figure 1. Schematic of the experimental setup. The 30 ps $355 \mathrm{~nm}$ beam from the Continuum PY-61C laser is split, attenuated, apertured, delayed, and then recombined before being moderately focused on the MALDI sample inside the vacuum system. The photodiode is used both to trigger acquisition of the mass spectrum and to measure the laser pulse energy.

width. The laser ran at a $20 \mathrm{~Hz}$ repetition rate, a shutter was used to select pulses from this train at $2 \mathrm{~Hz}$. After splitting, the two beams passed through separate attenuators for independent intensity adjustment.

The delayed beam was reflected from a corner cube (Laser Components, HRR-25, Olching, Germany) mounted on an optical rail of $2.5 \mathrm{~m}$ length (Linos X-95, Göttingen, Germany). Slight nonlinearities in the rail were compensated by mounting the corner cube on an $x-y$ micrometer stage and displacing it at each premeasured delay so as to return the beam to the same position. It was recombined with the reference beam via a second dielectric beam splitter. In this manner, the two beams could be made highly collinear.

The beams were somewhat defocused on the sample, so as to probe a larger area and thereby obtain a more stable signal. The sample spot was estimated to be roughly $0.5 \mathrm{~mm}$ in diameter. The delayed beam was apertured to a smaller diameter than the reference beam, so that it would remain within the reference beam spot in the event of minor misadjustments. The overlap was frequently checked by inserting a mirror after the lens and observing the spots on fluorescent paper with a magnifying glass. Fine tuning at the $\Delta t=0$ position before each measurement gave a clear optimum in the spatial overlap. The pulse energies were also measured after all delay optics and at various delays, to ensure that there was no change due to any geometric factors, such as clipping on apertures. The pulse energies at this point were up to $70 \mu \mathrm{J}$ per pulse, adjusted as necessary for individual samples.

The $\Delta t=0$ position was located using stimulated Raman scattering in both fused silica and toluene. The setup was identical to that used for the MALDI measurements, except that the final turning mirror was removed and the Raman medium placed at the focus. A photodiode mounted off the axis of the emerging beams was used to observe the Raman emission, at about $50 \mathrm{~cm}$ from the focal point. The zero point delay resolution was about $2 \mathrm{~mm}$ or $13 \mathrm{ps}$, and the stimulated Raman autocorrelated $355 \mathrm{~nm}$ pulse width was found to be $30 \pm 5$ ps.

The mass spectrometer was a lab-built instrument with a 2 $\mathrm{m}$ linear flight tube. A static rather than pulsed acceleration field was used. Ions were detected with a pair of microchannel plates, and the signal acquired on a $1 \mathrm{GS} / \mathrm{s}$ LeCroy 9350C (Chestnut Ridge, NY) digital oscilloscope. Each waveform was passed to a personal computer for further processing. The acquisition was triggered by the laser pulse via a photodiode. This signal was also acquired by a second digital oscilloscope and used for the intensity gating noted below.
It was necessary to have long-lasting and reasonably stable MALDI ion signals over a large number of laser shots, to cover several delays in each measurement series. A variety of sample preparation methods were tried, including dried droplets, electrospray, and pressed pellets. The latter was found to be superior for 2,5 dihydroxybenzoic acid (DHB, Fluka, Switzerland). About 30-40 mg of matrix was placed in a $1 \mathrm{~cm}$ diameter IR pellet press, spread evenly, and pressed at 5 tons for $30 \mathrm{~s}$. This resulted in a cohesive pellet of about $0.1 \mathrm{~mm}$ thickness. A piece of the pellet was broken off and affixed to the MALDI sample holder with double-sided tape.

Despite efforts to enhance signal stability, normal MALDI and laser fluctuations led to serious difficulties in obtaining reliable ion intensity measurements at multiple delay times. To lessen the effects of laser intensity drift, the measurement software was programmed (for most series) to accept only shots within a predetermined energy range. To account for change of MALDI signal strength as a sample was depleted, or due to any other effects, each delay time measurement was bracketed by measurements at zero delay. In this way, each delay was referenced to the most comparable $\Delta t=0$ values possible.

All major positive matrix ion signals $\left(\mathrm{M}-\mathrm{OH}^{+}, \mathrm{M}^{+}, \mathrm{MH}^{+}\right.$, and $\mathrm{MNa}^{+}$) were integrated and summed in software, for each laser shot. Shots were acquired individually, not averaged, so as to discriminate on the basis of pulse energy, as noted above. Each delay was measured for 50-100 laser shots, as were the bracketing $\Delta t=0$ measurements. The first measurements of each series were of the reference and delayed beams alone, to ensure that each remained below the ion generation threshold.

After each series, the sample was rotated to expose a fresh spot. After a measurement series, craters could be observed in the sample surface using an optical microscope $(66 \times$ magnification), but the depths were only a fraction of the thickness of the sample. The samples were typically conditioned for 50100 shots before a measurement series because in this time the ion generation threshold sometimes dropped slightly. The signal sometimes decreased or fluctuated significantly after several hundred shots. When this was obviously the case, the measurement series was terminated. The matrix ion signal for each delay was averaged and referenced to the average of the bracketing $\Delta t=0$ measurements.

\section{Results and Discussion}

An example 2PM mass spectrum is shown in Figure 2. The 2-pulse signal is strong, while that from the individual pulses is negligible. The method is, therefore, well suited to observing the time evolution of ionization processes in a MALDI sample.

The stability of the matrix ion signals when using samples prepared as described above is shown in Figure 3. Each point in the Figure represents the integrated matrix signal from a single laser shot $(\Delta t=0)$, no averaging was performed. As is typical in MALDI, different spots on a sample or different samples can exhibit distinctly different behaviors. For the spots shown in Figure 3, varying degrees of short- and long-term stability are apparent. In addition, not all spots or samples were stable for 1000 shots and sometimes showed a slow decay or a sudden drop after some hundreds of shots. Using the pressed pellet preparation method, different samples generally gave roughly comparable signal intensities. The four samples of Figure 3 varied by only about $15 \%$ in average signal level.

If MALDI ion formation is a single-molecule $1+1$ sequential two-photon process, then the 2PM curve should follow the (normally exponential) decay of the intermediate state involved. When the second pulse arrives, it finds less and less of the 


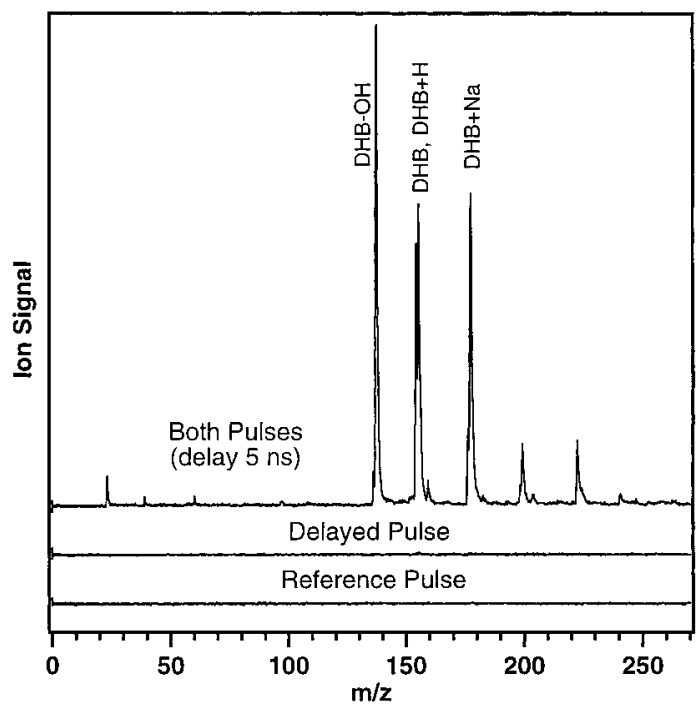

Figure 2. Two-pulse MALDI mass spectra using the reference and delayed pulses alone and both pulses together with 5 ns delay.

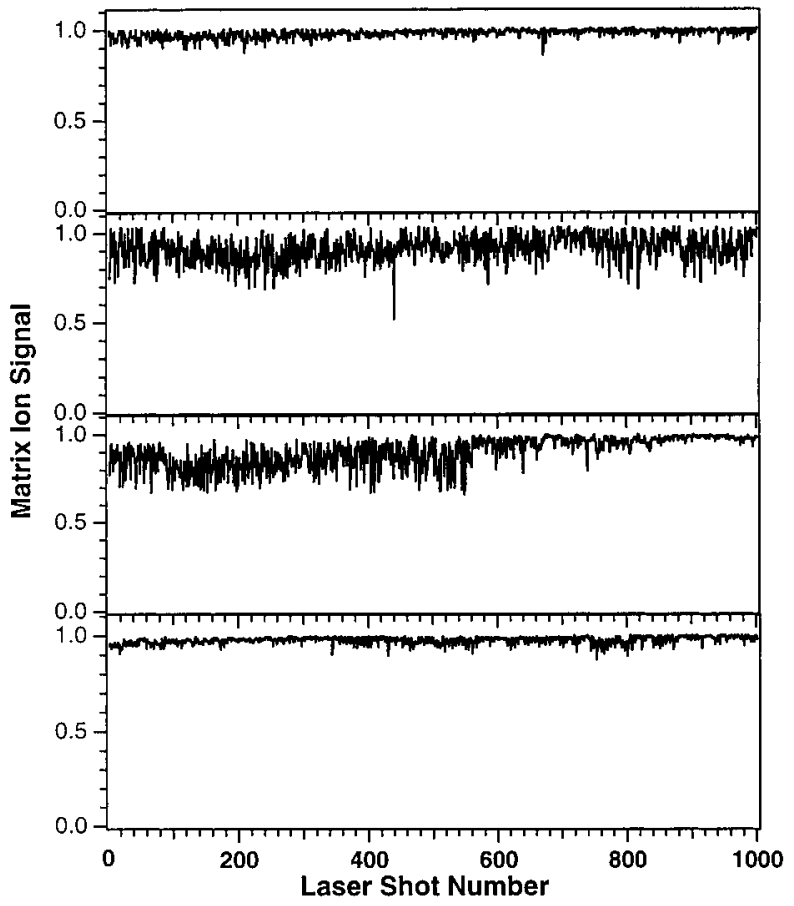

Figure 3. Integrated 2,5 DHB matrix ion intensity over 1000 laser shots for four different samples. The signal for each shot is shown, with no averaging or smoothing. The samples were prepared by pressing DHB powder in an IR pellet press at 5 tons for $30 \mathrm{~s}$.

critical intermediate as the delay time increases. This was the form of curve previously observed for analyte ions at lower time resolution in 2,5 DHB matrix. ${ }^{11}$ The decay also appeared to have about the same time constant as the solid-state fluorescence, suggesting that the fluorescent excited state was also the intermediate for MALDI ionization.

On the other hand, if MALDI ionization involves multiple centers, evolution of intermediates into new states, or migration of excitations in the crystal, then a more complex, nonexponential, 2PM curve should be observed. The maximum in ion generation may be displaced to some nonzero delay if, for example, the rate of conversion from the photoprepared state to a subsequent, more photoreactive intermediate state is not rapid.

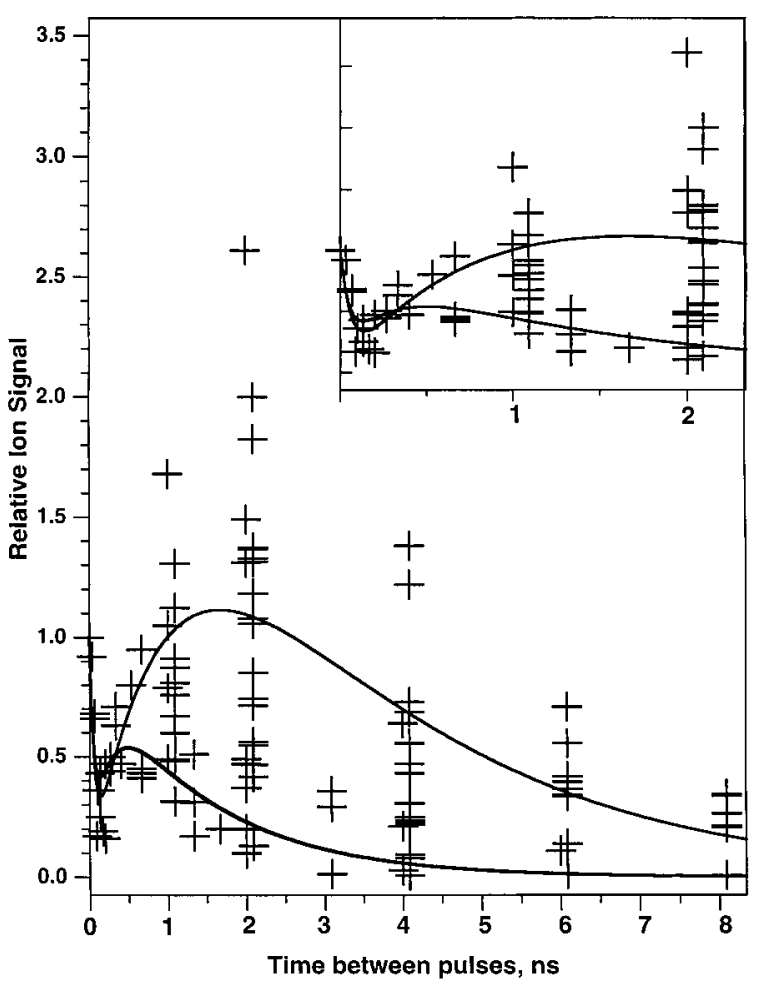

Figure 4. Integrated 2,5 DHB matrix ion signal vs delay between reference and delayed pulses. Each delay measurement was referenced to $\Delta t=0$ as described in the text. The zero-point delay uncertainty was 13 ps. The inset shows the short time region in more detail. The curves through the data schematically indicate the effect of larger or smaller contributions of the delayed ionization mechanism and are not fitted to the data.

As seen in Figure 4, both behaviors are observed for 2,5 DHB. Despite the scatter in the measurements, it is clear that the maximum in matrix ion generation generally occurs about 2 nanoseconds after the matrix has initially been irradiated. In addition, upon closer inspection of the short time region, a rapid decrease from $\Delta t=0$ is apparent, followed by a slower rise to the maximum. The minimum is about 0.5 , and the maximum about 2 times the $\Delta t=0$ signal, for a total dynamic range of a factor of 4 . After the maximum, the 2PM signal undergoes a roughly exponential decay.

Some samples show a strong maximum at $\Delta t=2 \mathrm{~ns}$, whereas others appear to exhibit a faster and weaker approach to the maximum, as well as an earlier decay. This is the cause of the apparent data scatter, particularly in the $1-2 \mathrm{~ns}$ region, and is believed to be a reflection of the empirically well-known "sweet spot" phenomenon in MALDI, and not simple experimental noise. Different samples or spots on a sample can give widely varying signal levels. This would appear to be connected to the efficiency of the delayed ionization mechanism. It should be noted that very few samples were sufficiently long lasting and stable to be measured over the full delay range, so the figure is a composite of a large number of measurement series. Further measurements with carefully purified matrix could determine if this effect is due to quenching impurities or is associated with local matrix consistency or morphology.

The schematic curves in Figure 4 are meant to suggest different relative strengths of the delayed ionization mechanism and are not in any way fitted to the data. For the upper curve, the rapid decay has a time constant of $65 \mathrm{ps}$, the rise $2.7 \mathrm{~ns}$, and the final decay $1.1 \mathrm{~ns}$. The latter two values were used together as the time constants in the expression for a sequential two-step reaction $\mathrm{A} \rightarrow \mathrm{B} \rightarrow \mathrm{C}$. In this case, $\mathrm{A}$ is the initially 
prepared excited state, $\mathrm{B}$ is the excited state in the nascent expanding plume from which ions are efficiently created by the second pulse, and $\mathrm{C}$ is the regenerated ground state

$$
I=\mathrm{e}^{-t / \tau_{1}}+\left(\left(K / \tau_{2}\right) /\left(1 / \tau_{3}+1 / \tau_{2}\right)\right)\left(\mathrm{e}^{-t / \tau_{2}}-\mathrm{e}^{-t / \tau_{3}}\right)
$$

These states are not necessarily single molecule states but may be collective excitations (excitons). In the lower curve, the second and third time constants were 1.5 and $0.2 \mathrm{~ns}$, respectively, to qualitatively represent a more strongly quenched ion formation process.

The initial drop in 2PM signal shows that a fast ionization mechanism is operative. However, because the time scale is possibly somewhat greater than the laser pulse width itself, this may not be limited to direct multiphoton processes. Its shorter time scale means that it is probably of limited importance compared to the slower multistep mechanism, at least at moderate laser powers. The latter profits from the longer allowable times between the arrival of subsequent photons, and thus, its integrated contribution over the typical nanosecond MALDI laser pulse will be greater.

There are numerous candidates for the intermediate step(s) leading to the rise in ion formation to a delayed maximum. These include single molecule intersystem crossing, exciton pooling, local non-Boltzman heating by internal conversion, and others. In light of the indications noted above for a delayed "gas phase" ion formation mechanism, it is interesting to consider this possibility in slightly more detail.

Measurements of initial matrix ion velocities cover a range of a few 100 to $2000 \mathrm{~m} / \mathrm{s}^{46-52}$ If we take $500-1000 \mathrm{~m} / \mathrm{s}$ as being approximately correct for 2,5 DHB, then the MALDI plume moves $1-2$ micrometer in 1 nanosecond (the acceleration time is expected to be very short ${ }^{5,6,13}$ ). The maximum in ion production observed in Figure 4 thus occurs after about 2 micrometers of expansion, assuming that the first pulse is efficient in inducing desorption, but not ionization. The present data therefore suggest that ion formation is most efficient when the laser interacts with a reasonably dense, but not solid material, which has also been energized by absorption of the first laser photons. Clusters may be numerous and very important during this time. ${ }^{18,25,30}$ The lower density may also be important in itself, for example due to a reduced ion quenching rate.

For comparison, energy deficits in time-of flight distributions suggest that matrix ions are created at distances up to 10-15 micrometers from the surface. ${ }^{39,41}$ This points in the same direction as the present measurements and probably is an overestimate because it does not account for the effects of surface roughness and, hence, ions formed at different potentials; nor does it differentiate between primary ionization or secondary plume reactions.

\section{Conclusions}

Time-delayed 2-pulse MALDI results are reported for 2,5 DHB matrix ions using $30 \mathrm{ps} 355 \mathrm{~nm}$ excitation. The data indicate that at least two ion-formation mechanisms are active. A rapidly decaying mechanism leads to a small number of ions, whereas a later one produces many ions, with a characteristic peak at about 2 nanoseconds. At longer times, ion production falls, as the excited states decay and the plume expands.

These results constrain models for the dominant 2,5 DHB MALDI ionization mechanism. These must include at least one intermediate and relatively slow step between absorption of the first photon and subsequent photon(s) leading to ion states. This step may either be unimolecular, excitonic, or correspond to the physical phase change.

On the basis of the time scales observed here, the currently favored nitrogen lasers with a 3 ns pulse width appear well adapted to this matrix material. Substantially longer or shorter laser pulses are not likely to be advantageous.

Acknowledgment. This work as supported by ETH internal research grant 0-20-402-97 and NSF Grant Number CHE9873610. The authors would like to thank Prof. Renato Zenobi for support and stimulating discussions, and Dr. Alan Joly for helpful discussions regarding stimulated Raman.

\section{References and Notes}

(1) Vertes, A.; Irinyi, G.; Gijbels, R. Anal. Chem. 1993, 65, 2389

(2) Bencsura, A.; Vertes, A. Chem. Phys. Lett. 1995, 247, 142.

(3) Balazs, L.; Gijbels, R.; Vertes, A. Anal. Chem. 1991, 63, 314.

(4) Bencsura, A.; Navale, V.; Sadeghi, M.; Vertes, A. Rapid Commun Mass Spectrom. 1997, 11, 679.

(5) Wu, X.; Sadeghi, M.; Vertes, A. J. Phys. Chem. B 1998, 102, 4770

(6) Zhigilei, L. V.; Garrison, B. J. Rapid Comm. Mass Spectrom. 1998 12,1273

(7) Zhigilei, L. V.; Kodali, P. B. S.; Garrison, B. J. J. Phys. Chem. B 1997, 101, 2028

(8) Zhigilei, L. V.; Garrison, B. J. Appl. Phys. Lett. 1997, 71, 551

(9) Johnson, R. E. Models for Matrix-Assisted Laser Desorption and Ionization: MALDI. In Large Ions: Their Vaporization, Detection and Structural Analysis; Baer, T., Ng, C. Y., Powis, I., Eds.; John Wiley and Sons: New York, 1996; p 49.

(10) Johnson, R. E. Int. J. Mass Spectrom. Ion Processes 1994, 139, 25

(11) Moskovets, E.; Sadeghi, M.; Bencsura, A.; Vertes, A. Proceedings of 47th ASMS Conference on Mass Spectrometry and Allied Topics, Dallas, Texas, 1999; p 769.

(12) Handschuh, M.; Nettesheim, S.; Zenobi, R. Appl. Surf. Sci. 1998, 137,125 .

(13) Zhigilei, L. V.; Garrison, B. J. Appl. Phys. A 1999, 69, S75.

(14) Zenobi, R.; Knochenmuss, R. Mass Spectrom. Rev. 1998, 17, 337.

(15) Mowry, C. D.; Johnston, M. V. J. Phys. Chem. 1994, 98, 1904

(16) Puretzky, A. A.; Geohegan, D. B. Chem. Phys. Lett. 1997, 286, 425 .

(17) McCarley, T. D.; McCarley, R. L.; Limbach, P. A. Anal. Chem 1998, 70, 4376

(18) Karbach, V.; Knochenmuss, R. Rapid Commun. Mass Spectrom. 1998, 12,968

(19) Knochenmuss, R.; Karbach, V.; Lin, Q. Proceedings of 47th ASMS Conference on Mass Spectrometry and Allied Topics, Dallas, Texas, 1999; p 1059.

(20) Karas, M.; Bachmann, D.; Bahr, U.; Hillenkamp, F. Int. J. Mass Spectrom. Ion Processes 1987, 78, 53.

(21) Krause, J.; Stoeckli, M.; Schlunegger, U. P. Rapid Commun. Mass Spectrom. 1996, 10, 1927.

(22) Gimon, M. E.; Preston, L. M.; Solouki, T.; White, M. A.; Russell, D. H. Org. Mass Spectrom. 1992, 27, 827.

(23) Chiarelli, M. P.; Sharkey, A. G.; Hercules, D. M. Anal. Chem. 1993, 65,307 .

(24) Knochenmuss, R.; Karbach, V.; Wiesli, U.; Breuker, K.; Zenobi, R. Rapid Commun. Mass Spectrom. 1998, 12, 529.

(25) Knochenmuss, R.; Dubois, F.; Dale, M. J.; Zenobi, R. Rapid Commun. Mass Spectrom. 1996, 10, 871.

(26) Breuker, K.; Knochenmuss, R.; Zenobi, R. Int. J. Mass Spectrom. 1999, 184,25 .

(27) Breuker, K.; Knochenmuss, R.; Zenobi, R. J. Am. Soc. Mass Spectrom. 1999, 10, 1111.

(28) Knochenmuss, R.; Stortelder, A.; Zenobi, R. J. Mass Spectrom., submitted for publication.

(29) Allwood, D. A.; Dyer, P. E.; Dreyfus, R. W. Rapid Commun. Mass Spectrom. 1997, 11, 499.

(30) Karas, M.; Glückmann, M.; Schäfer, J. J. Mass Spectrom. 2000, 35,1

(31) Breuker, K.; Knochenmuss, R.; Zenobi, R. J. Mass Spectrom., submitted for publication.

(32) Johnson, R. E.; Sundqvist, B. U. R. Rapid Commun. Mass Spectrom. 1991, 5, 574.

(33) Chevrier, M. R.; Cotter, R. J. Rapid Commun. Mass Spectrom. 1991, 5,611 .

(34) Beavis, R. C. Org. Mass Spectrom. 1992, 27, 864.

(35) Riahi, K.; Bolbach, G.; Brunot, A.; Breton, F.; Spiro, M.; Blais, J.-C. Rapid Commun. Mass Spectrom. 1994, 8, 242 
(36) Dreisewerd, K.; Schurenberg, M.; Karas, M.; Hillenkamp, F. Int J. Mass Spectrom. Ion Proc. 1996, 154, 171.

(37) Ens, W.; Schürenberg, M.; Hillenkamp, F. Proceedings of 45th ASMS Conference on Mass Spectrometry and Allied Topics, Palm Springs, California, 1997; p 1099

(38) Dreisewerd, K.; Schurenberg, M.; Karas, M.; Hillenkamp, F. Int. J. Mass Spectrom. Ion Proc. 1995, 141, 127.

(39) Zhou, J.; Ens, W.; Standing, K. G.; Verentchikov, A. Rapid Commun. Mass Spectrom. 1992, 6, 671.

(40) Kinsel, G. R.; Edmondson, R. D.; Russell, D. H. J. Mass Spectrom. 1997, 32, 714

(41) Kinsel, G. R.; Gimon-Kinsel, M. E.; Gillig, K. J.; Russell, D. H. J. Mass Spectrom. 1999, 34, 684.

(42) Demirev, P.; Westman, A.; Reimann, C. T.; Håkansson, P.; Barofsky, D.; Sundqvist, B. U. R.; Cheng, Y. D.; Seibt, W.; K, S. Rapid Commun. Mass Spectrom. 1992, 6, 187.

(43) Tang, X.; Sadeghi, M.; Olumee, Z.; Vertes, A. Rapid Commun. Mass Spectrom. 1997, 11, 484.
(44) Ehring, H.; Sundqvist, B. U. R. J. Mass Spectrom. 1995, 30, 1303

(45) Ehring, H.; Sundqvist, B. U. R. Appl. Surf. Sci. 1996, 96-8, 577.

(46) Beavis, R. C.; Chait, B. T. Chem. Phys. Lett. 1991, 181, 479.

(47) Huth-Fehre, T.; Becker, C. H. Rapid Commun. Mass Spectrom. 1991, 5, 378 .

(48) Pan, Y.; Cotter, R. J. Org. Mass Spectrom. 1992, 27, 3.

(49) Spengler, B.; Bökelmann, V. Nucl. Instr. Methods Phys. Res., Sect. $B$ 1993, 82, 379.

(50) Juhasz, P.; Vestal, M. L.; Martin, S. A. J. Am. Soc. Mass Spectrom 1997, 8, 209.

(51) Dworschak, R. G.; Spicer, V.; Ens, W.; Standing, K. G. Proceedings of 46th ASMS Conference on Mass Spectrometry and Allied Topics, Orlando, Florida, 1998; p 932.

(52) Glückmann, M.; Karas, M. Proceedings of 47th ASMS Conference on Mass Spectrometry and Allied Topics, Dallas, Texas, 1999; p 2232. 